\title{
Cooperation between Human DAF and CD59 in Protecting Cells from Human Complement-mediated Lysis
}

\author{
Li Xu, Wenlan Wu, Zhouzhou Zhao, Huanjie Shao, Wanhong Liu, Hui Liu and Wenxin Li* \\ State Key Laboratory of Virology, College of Life Sciences, Wuhan University, Wuhan 430072, Hubei Province, P. R. China
}

Received 23 March 2005, Accepted 25 July 2006

The complement $(C)$ regulatory proteins decay accelerating factor (DAF, CD55) and CD59 could protect host cells using different mechanisms from $\mathrm{C}$-mediated damage at two distinct levels within the $\mathrm{C}$ pathway. Co-expression of DAF and CD59 would be an effective strategy to help overcome host $\mathrm{C}$-induced xenograft hyperacute rejection. In this study, we made a construct of recombinant expression vector containing DAF and CD59 CDNA and the stable cell lines were obtained by G418 selection. Extraneous genes integration and co-expression were identified by PCR, RT-PCR and Western blot analysis. Human c-mediated cytolysis assays showed that NIH/3T3 cells transfected stably with pcDNA3-CD59, pcDNA3DAF, and pcDNA3-CD59DAF-DP were protected from Cmediated damage and that synchronously expressed human CD59 and DAF provided the most excellent protection for host cells as compared with either human CD59 or DAF expressed alone. Therefore, the construct represents an effective and efficacy strategy to overcome C-mediated damage in cells and, ultimately, in animals.

Keywords: Complement regulatory proteins, Co-expression, Cooperation, Hyperacute rejection

\section{Introduction}

Xenotransplantation, as an alternative approach for patients with end stage organ failure, has attracted more and more attention because of the serious shortage of human organs for allotransplantation (Lambrigts et al., 1998). However, progress towards its clinical application has been hampered by the phenomenon of hyperacute rejection (HAR), which occurs

\footnotetext{
*To whom correspondence should be addressed. Tel: 86-027-68752831; Fax: 86-027-68752146

E-mail: liwxlab@whu.edu.cn
}

almost immediately upon reperfusion of the transplanted organ and leads dramatic and irreversible graft destruction within minutes to hours (Calne et al., 1970; Platt et al., 1991). The activation of complement is one of critical events in the pathogenesis of HAR. Human complement regulatory proteins (hCRPs), such as decay accelerating factor (DAF), protectin CD59 etc., have been studied intensively in order to help overcome C-mediated HAR in Xenotransplantation (XNS) (Brooimans et al., 1992; Byrne et al., 1995; Nagahama et al., 2000; 2001; 2002; Huang et al., 2001; Shiraishi et al., 2002). DAF is a glycosyl phosphatidylinositol (GPI)-anchored membrane regulator of complement that inhibits the $\mathrm{C} 3$ convertases of both the classical and alternative pathways (Nicholson-Weller et al., 1982). CD59, as well as DAF, is a GPI-anchored membrane glycoprotein, which directly regulates the formation and function of the terminal cytolytic membrane attack complex (MAC) (Meri et al., 1990; Rollins et al., 1990). Transgenic animals expressing hCRPs, such as CD59, or DAF, have been developed (Fodor et al., 1994; Byrne et al., 1995; 1997; Diamond et al., 1996; Cowan et al., 2000; Cozzi et al., 2000; Lavitrano et al., 2002; Menoret et al., 2004). These transgenic animals expressing hCRPs could provide excellent protection from human serum complement attack, and these transgenic organs survived longer than nontransgenic control organs in vivo transplantation experiment. However, sufficient protection from the massive XNS reactivity was not achieved so far. And recent studies have suggested that double hCRPs exhibited more protection against cytolysis by human serum compared to the cells with only one expressed alone (Byrne et al., 1997; Chen et al., 1999; Cowan et al., 2000; Huang et al., 2001; Nagahama et al., 2000; 2001). So an approach enabling the synchronous expression of multiple hCRPs in porcine tissue should be developed. In this study, a recombinant expression vector pcDNA3-CD59DAF-DP was constructed in order to coexpress hDAF and hCD59 in NIH/3T3 cells. We demonstrate that co-expression of the two hCRPs could provide excellent protection against $\mathrm{C}$-mediated cytolysis by their synergistic action. 


\section{Materials and Methods}

Construction of expression plasmid. The whole length cDNA encoding human DAF was amplified by PCR using 3-week-old human embryo cDNA library as templates (Gou et al., 1999). The full-length cDNA was subcloned into a mammalian expression vector pcDNA3 between a CMV promoter and a BGH-polyadenylated sequence (Invitrogen). DAF cDNA inserted into the XhoI-ApaI site of pcDNA3 was called pcDNA3-DAF. Sequence of the hDAF gene was confirmed by DNA sequencing. Restriction enzymes were from Takara Biotechnology Co., Ltd.

The $1.46 \mathrm{~kb}$ long fragment containing CD59 cDNA, CMV promoter and BGH-polyadenylated was obtained by PCR using the plasmids pcDNA3-CD59 (Huang et al., 2001) as a substrate, and then it was subcloned into the $B g / I I$ site of the pcDNA3-DAF. The two plasmids, pcDNA3-CD59DAF (cis) and pcDNA3-CD59DAF (trans) were transformed into Escherichia coli $\mathrm{DH} 5 \alpha$ cells and isolated, characterized and amplified by using standard techniques. The vector pcDNA3-CD59DAF (trans) was named pcDNA3CD59DAF-DP (Fig.1A).

Cell culture and transfection. NIH/3T3 cells (mouse fibroblasts; Chinese center Type Culture Collection, Wuhan, P.R. China) were cultured in Dulbeco's modified Eagle's medium (DMEM) supplemented with $10 \%$ heat-inactivated fetal calf serum (FCS). Cultures were maintained in $5 \% \mathrm{CO}_{2}$ at $37^{\circ} \mathrm{C}$. D-hank's containing $0.02 \%(\mathrm{w} / \mathrm{v})$ EDTA-2Na and $0.125 \%(\mathrm{w} / \mathrm{v})$ trypsin (Sigma) were used to detach the cells.

A total of $5 \times 10^{5} \mathrm{NIH} / 3 \mathrm{~T} 3$ cells on a $60 \mathrm{~mm}$ diameter tissue culture plate were transfected with $8.8 \mu \mathrm{g}$ of plasmid pcDNA3CD59DAF-DP DNA by calcium phosphate precipitation method as reported by Sambrook et al (Sambrook et al., 1989). Transfected $\mathrm{NIH} / 3 \mathrm{~T} 3$ cells were incubated for $24 \mathrm{hr}$ in DMEM, 10\% FCS, in $5 \% \mathrm{CO}_{2}$ at $37^{\circ} \mathrm{C}$, after which $400 \mu \mathrm{g} / \mathrm{ml} \mathrm{G} 418$ (Gibco BRL) was added and selection carried out over 3-5 wk. Surviving clones were expanded in tissue culture plates and flasks. To verify the stable integration of the two genes, the total genomic DNA were extracted from the transfected clones and used as templates for PCR.

Isolation of total RNA and analysis of hDAF and hCD59 coexpression by RT-PCR. Expression of the double genes was evaluated by RT-PCR from the total RNA samples of pcDNA3CD59DAF-DP NIH/3T3 transfectants. Total RNA was isolated from transfected NIH/3T3 cells by using Trizol ${ }^{\circledR}$ LS Reagent (Gibco BRL) according to the manufacturer's instructions and treated with RNase-free DNase I (Promega). First strand cDNA was prepared by reverse transcriptase (Gibco BRL). A 1336-bp fragment was amplified to detect the expression of hDAF using 5'CGC CTC GAG CGT CCT TGT TCT AAC CCG-3' as forward primer and 5'-ACT GGG CCC TTG CTC TGT TGA CAT TCC-3' as reverse primer, and a 420-bp fragment of hCD59 was detected using 5'-CCG AAT TCT GTG GAC AAT CAC AAT GG-3' as forward primer and 5'-CTG GTC TAG ACT TAG GGA TGA AGG C-3' as reverse primer.

Western blot analysis. To analyze the expression of human DAF and CD59 proteins on the transfected NIH/3T3 cells, monolayers of pcDNA3 (negative control) and pcDNA3-CD59DAF-DP transfetants were lysed by addition of lysis buffer containing $100 \mathrm{mM} \mathrm{NaCl}$, $10 \mathrm{mM}$ Tris-HCl (PH 8.0), 1 mM EDTA (pH 8.0), 1\% Triton X100 and $1 \mathrm{mM}$ protease inhibitor: phenyl-methylsulfonyl fluoride (PMSF, Sigma).

The lysates were run on $12 \%$ polyacrylamide gel using the method of Laemmli (Laemmli et al., 1970), and transferred onto nitrocellulose membrane (Millipore, Bedford, MA) in $25 \mathrm{mM}$ Tris, $192 \mathrm{mM}$ glycine, and 20\% (v/v) methanol, $\mathrm{pH} 8.3$, using a Transblot apparatus (Bio-Rad, Hercules, CA) for $1 \mathrm{hr}$ at $300 \mathrm{~mA}$ according to the method of Towbin et al (Towbin et al., 1979). The membranes were blocked with $5 \%(\mathrm{w} / \mathrm{v})$ non-fat dried milk in Trisbuffered saline (TTBS, $10 \mathrm{mM}$ Tris, $0.15 \mathrm{M} \mathrm{NaCl}, 0.1 \%$ (v/v) Tween 20), incubated with goat polyclonal antibody CD59 (c-19): sc-7076 (Santa Cruz Biotechnology, Inc.) or polyclonal goat antihuman CD55 antibody (c-20): sc-7064 (Santa Cruz Biotechnology, Inc.) in a dilution of $1 / 100$ in TTBS for $2 \mathrm{hr}$ at $37^{\circ} \mathrm{C}$, washed with TTBS and then incubated for $1 \mathrm{hr}$ with horseradish peroxidaseconjugated rabbit anti-goat Ig (Zhongshan Biotechnology) at room temperature. The blots were developed in diaminobenzidine (DAB, Sigma) and hydrogen peroxide.

Human complement-mediated cytolysis assay. Normal human serum (NHS) to be used as a complement source was obtained from the blood of healthy volunteers in the laboratory and stored in aliquots at $-80^{\circ} \mathrm{C}$. C-mediated cytolysis assays were performed as described previously (Huang et al., 2001). Briefly, an equal volume $(100 \mu \mathrm{l})$ of the NIH/3T3 transfectants were incubated in serial dilutions (neat, $1 / 2,1 / 4,1 / 8$, and 1/16) of NHS for $1 \mathrm{hr}$ at $37^{\circ} \mathrm{C}$, after which $\mathrm{C}$-mediated cytolysis was determined by microscopic examination following trypan blue staining. Cell viability was determined by trypan blue exclusion and the percentage cytotoxicity was calculated. The 3-[4,5-dimethylthiazol-2-yl]-2,5-diphenyl tetrazolium bromide (MTT) assay was also used to determine the cellular damage of NIH/3T3 caused by human complement activation (Nagahama et al., 2002).

To further investigate their synergistic action, blockade of hCD59 or hDAF function was carried out by addition of the anti-hCD59 mouse monoclonal antibody (mAb), BRIC229 or anti-hDAF mouse mAb, BRIC216 (IBGRL,UK) in Ab blocking experiments. For measurement of cytotoxicity, each condition was performed in triplicate and each experiment was performed three times. Noserum-added cell death was approximately $5 \%$ in all assays and was subtracted as the background. The data shown are the mean of three separate experiments using the same quantity of antibody.

\section{Results}

Transfection of NIH/3T3 cells with pcDNA3-CD59DAFDP vector. A mammalian expression vector was constructed, which contains the neomycin resistance gene and the two independent $\mathrm{CMV}$ promoters controlling the expression of human DAF and CD59 cDNA. The double genes were coexpressed in NIH/3T3 cells by transfection, followed by selection of permanent transfectants in the presence of G418. Stable cell lines were generated by selection. As shown in Fig. $1 \mathrm{~B}$, integration of the two genes in the chromosomal DNA in transfected cells was ascertained by a CD59-specific 420 bp 
(A)

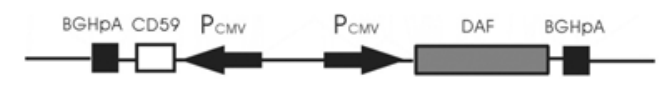

(B)
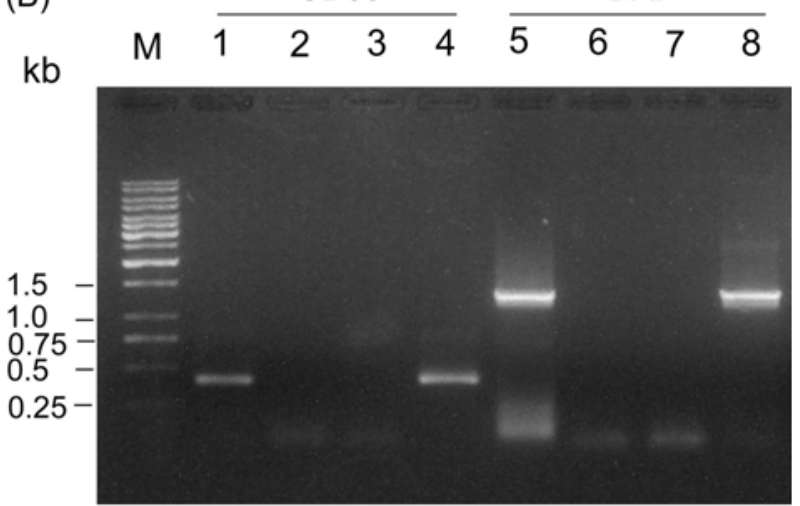

Fig. 1. Strategy for co-expressing of hCD59 and hDAF in stable transfected NIH/3T3 cells. (A) Schematic diagram of the expression vector pcDNA3-CD59DAF-DP used for this study. The cDNAs for hCD59 and hDAF were inserted into the multiple cloning site of pcDNA3 vector under control the CMV promoters and correct orientation of insert (trans) was confirmed by using standard techniques. Transcription from the CMV promoter into the hCD59 gene is in the opposite direction from transcription that initiates transcription hDAF by the other CMV promoters. (B) PCR analysis of the integration of hCD59 and hDAF genes on the stable transfected NIH/3T3 cells. The primers of the hCD59 gene were used in lanes 1-4 and the primers of hDAF gene were used in lanes 5-8. Templates: lanes 1,5, high-molecular-mass DNA of NIH/3T3 pcDNA3-CD59DAFDP; lanes 2,6, high-molecular-mass DNA of NIH/3T3 pcDNA3 cells; Lanes 4,8, plasmid pcDNA3-CD59DAF-DP; lanes 3,7, high-molecular-mass DNA of NIH/3T3 cells; $\mathrm{M}, 1 \mathrm{~Kb}$ DNA ladder marker.

(Fig. 1B, lane 1) and a DAF-specific 1336bp (Fig. 1B, lane 5) DNA fragment, when the total genomic DNA of NIH/3T3 cells transfected with pcDNA3-CD59DAF-DP was used as templates. In contrast, nonspecific amplification was detected when the total genomic DNA of NIH/3T3 pcDNA3 cells (Fig.1B, lanes 2,6) or NIH/3T3 (Fig. 1B, lanes 3, 7) was the template.

Expression analysis. RT-PCR was carried out to ascertain the co-expression of hDAF and hCD59 mRNA in the stable pcDNA3-CD59DAF-DP transfectants with primers specific for the two genes. In Fig. 2, a 420 bp DNA fragement specific for hCD59 (Fig. 2, lane 1) and $1330 \mathrm{bp}$ DNA products specific for hDAF (Fig. 2, lane 5) were detected in the transfectants pcDNA3-CD59DAF-DP. No specific PCR products were observed when RNA of pcDNA3-CD59DAF-DP transfectants (Fig. 2, lanes 2,6) and cDNA of pcDNA3 transfected cells (Fig. 2, lanes 3,7) were used as templates.

In order to further explore the pattern of co-expression of the double genes, Western blot analysis of cell lysates from pcDNA3-CD59DAF-DP transfectants was performed utilizing

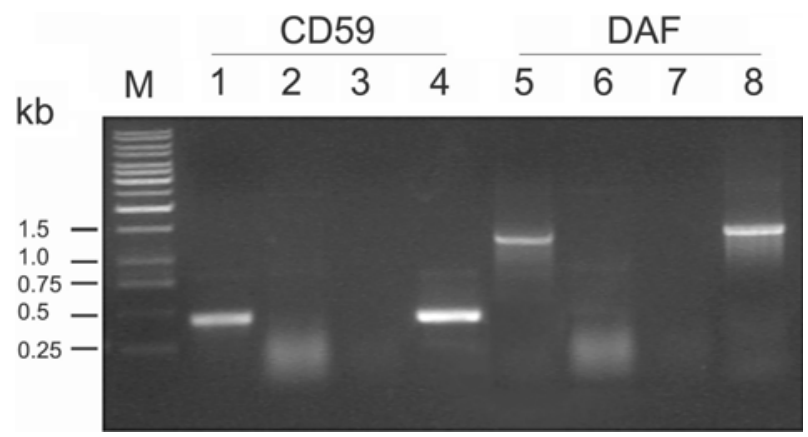

Fig. 2. RT-PCR analysis of human CD59 and DAF genes coexpression in pcDNA3-CD59DAF-DP transfected cell line. The primers for the CD59 gene were used in lanes 1-4 and the primers for DAF gene was used in lanes 5-8. Templates: lanes 1, 5, cDNA of NIH/3T3 pcDNA3-CD59DAF-DP transfectants; lanes, 2,6, total RNA from NIH/3T3 pcDNA3-CD59DAF-DP transfectants; lanes, 3,7, cDNA of NIH/3T3 pcDNA3 transfectants; lanes, 4,8, DNA of the plasmid pcDNA3-CD59DAF-DP as positive controls; $\mathrm{M}, 1 \mathrm{~Kb}$ DNA ladder marker

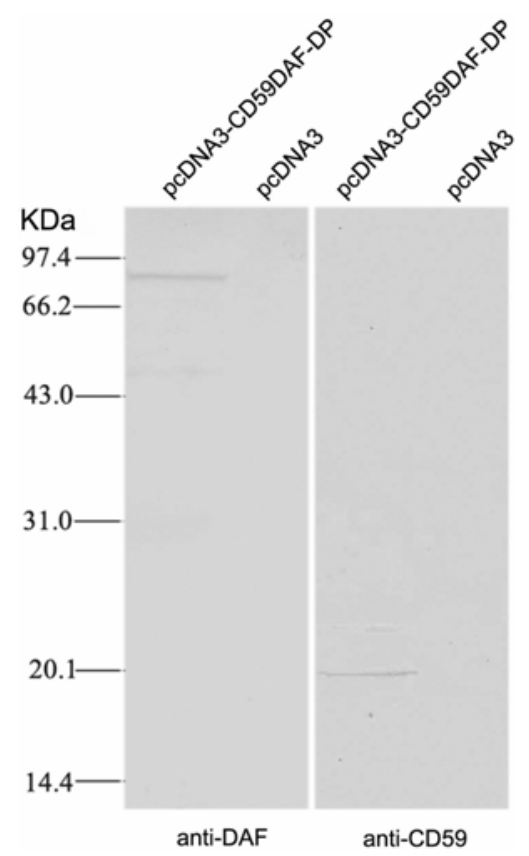

Fig. 3. Western blot analysis of human DAF and CD59 proteins detected by polyclonal antibodies to human CD59 and DAF on the pcDNA3-CD59DAF-DP transfectants. The position of molecular weight markers is indicated.

specific goat polyantibodies against $\mathrm{hDAF}$ and hCD59. As shown in Fig. 3, human CD59 and DAF migrated as the expected bands of $20-$ and $75-\mathrm{kDa}$ bands, respectively. In contrast, no specific bands were observed in the vector control-transfected cells. These results demonstrated that hDAF and hCD59 molecules were both expressed on the pcDNA3-CD59DAF-DP transfectants. 


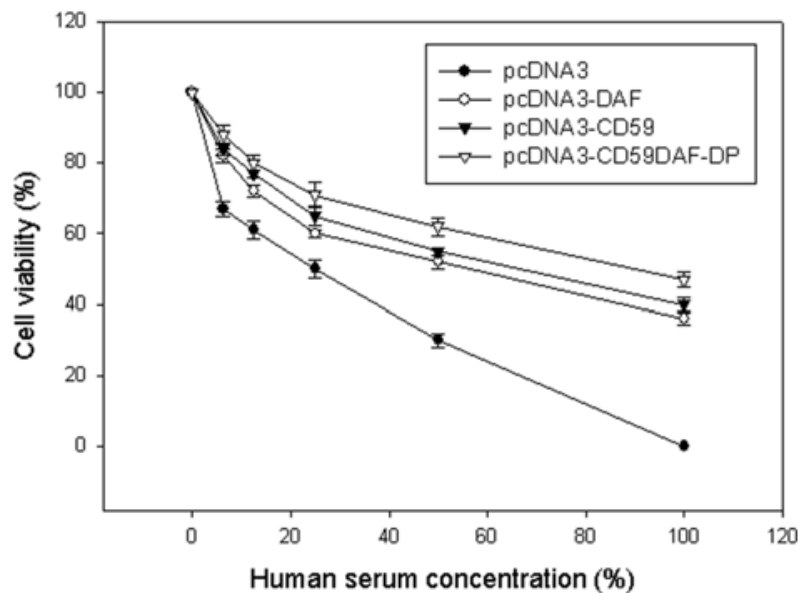

Fig. 4. Protection of NIH/3T3 cells from C-mediated damage by transfected molecules. Control NIH/3T3 cells (pcDNA3 vector only) and transfectants (pcDNA3-CD59, pcDNA3-DAF and pcDNA3-CD59DAF-DP) were incubated with increasing concentrations of human serum for $1 \mathrm{hr}$ at $37^{\circ} \mathrm{C}$. Viabilities were determined by dye exclusion with trypan blue and the percentage cytotoxicity was calculated. Results represent means of three independent experiments and error bars represent the standard deviation.

Protection of murine cells from human complementmediated cytolysis by cooperation between human DAF and CD59. To assess the efficacy of our combinatorial genetic approach in NIH/3T3 cells, their protective function was measured by comparing the ability against $\mathrm{C}$-mediated cytotoxicity when being challenged with human serum. The four transfectants, NIH/3T3 pcDNA3 cells, NIH/3T3 pcDNA3CD59 cells, NIH/3T3 pcDNA3-DAF cells, and NIH/3T3 pcDNA3-CD59DAF-DP cells, were incubated with increasing dilutions of human serum and cell cytotoxicity was assessed by trypan blue staining. A protective effect was observed as shown in Fig. 4. Under identical conditions, the control NIH/ $3 \mathrm{~T} 3$ pcDNA3 cells were effectively lysed by human serum in a dose dependent manner. However NIH/3T3 cells transfected with pcDNA3-CD59, pcDNA3-DAF and pcDNA3-CD59DAFDP showed resistance to human complement attack. The pcDNA3-CD59DAF-DP transfectants showed the most significant protection from human serum-mediated cytolysis by the cooperative inhibition of hDAF and hCD59 proteins from $6.25 \%$ to $100 \%$ serum. The cytolysis was suppressed in three transfectants: $36 \%, 40 \%, 47 \%$ were reduced in the three transfectants, respectively, compared with control vector transfectants on exposure to $100 \%$ NHS. MTT assay was also performed with monolayer NIH/3T3 cultured in 96-well microplates and the results were consistent with those obtained by trypan blue assay (data not shown).

Furthermore, to evaluate the functional advantage of CD59DAF over DAF or CD59, Ab blocking experiments were performed. As shown in Fig. 5, preincubation with anti-DAF or anti-CD59 antibodies decreased their protection against complement attack and enhanced their susceptibility to C-

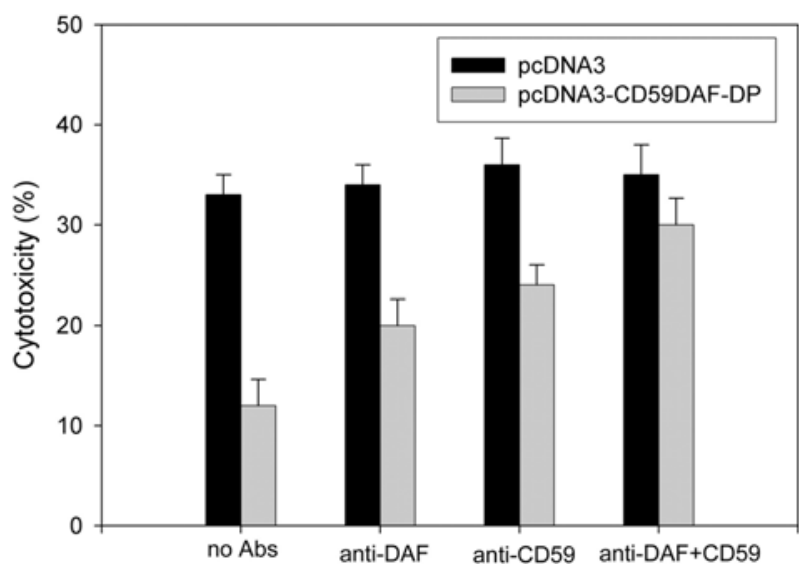

Fig. 5. Antibody-blocking experiments were performed on the pcDNA3 and pcDNA3-CD59DAF-DP transfectants. The transfected cells were treated with mouse anti-DAF, anti-CD59 monoclonal antibodies alone or in combination to block the function of CD59 and DAF. Subsequently, these cells were incubated with $6.25 \%$ normal human serum. Results are means of three independent experiments and error bars represent the standard deviation.

mediated lysis. Blocking of CD59 on the surface of pcDNA3CD59DAF-DP transfectants rendered these cells more susceptible to complement attack as compared to that blocked with anti-DAF antibodies. A combination of both antibodies led a thorough lysis: cellular death rates increased from $12 \%$ to $30 \%$ when pcDNA3-CD59DAF-DP transfectants were treated with $6.25 \%$ NHS. In contrast, the monoclonal antibodies had no effect on the pcDNA3 transfectants. The present data suggest that these two membrane proteins could act synergistically and convey a functional cooperation between DAF and CD59 molecules in protection non-human cells from C-mediated damage.

\section{Discussion}

Human membrane complement regulatory proteins (hCRPs) are known to be important for protecting host cells against homologous C attack. Moreover, hCRPs, such as Decayaccelerating factor (DAF), membrane cofactor protein (MCP) and CD59 act cooperatively at different levels in the complement activation cascade (Nagahama et al., 2002). Therefore, there is an interest in developing membrane complement proteins as candidate molecules for therapy of disease states in which complement activation and deposition is involved in pathogenesis. In this context, a number of attempts have been made to induce non-human cells to express high level of hCRPs (Cowan et al., 2000; Cozzi et al., 2000), which may successfully confer resistance to human C-mediated lysis on the non-human cells and overcome the first major barrier against xenotransplantation. So far, the major approach to overcome HAR is the use of organs from genetically 
engineered pigs transgenic for human complement regulatory proteins. Genetically engineered pigs that expressed human complement regulatory proteins have been reported by several groups (Fodor et al., 1994; Byrne et al., 1995; 1997; Diamond et al., 1996; Cowan et al., 2000; Cozzi et al., 2000; Lavitrano et al., 2002; Menoret et al., 2004). These reports demonstrated that hCD59 and hDAF can be expressed in other species without detriment and that expression of these molecules efficiently protects host cells from C-mediated damage. Transgenic pigs expressing more than one type of hCRPs were produced by intercrossing single transgenic pigs (Byrne et al., 1997; Chen et al., 1999) or by co-microinjection of gene constructs for hCRPs (Cowan et al., 2000; Menoret et al., 2004). Previously, a CD46-CD55 chimeric complement regulatory was developed and shown to have both membrane cofactor and decay accelerating activities (Iwata et al., 1994; Brodbeck et al., 2000). The DC (NH2-CD59-DAF-GPI) chimeric molecule was also made, which expressed both DAF and CD59 activity, thereby regulating C3 convertase and MAC assembly (Fodor et al., 1995). However, a vector utilizing two independent promoters for co-expression of multiple genes of hCRPs in its native configuration by nonviral system, has never been reported and would be advantageous as a potential therapeutic strategy to inhibit Cmediated hyperacute rejection during xenotransplantation.

In this study, we constructed a recombinant vector pcDNA3CD59DAF-DP expressing multiple therapeutic genes (DAF and CD59) simultaneously in order to establish a suitable construct for xenotransplantation. We demonstrated that transfected cells with pcDNA3-CD59DAF-DP co-expressed human CD59 and DAF at both the mRNA and protein levels by using RT-PCR and Western blot analysis. The functional studies revealed that NIH/3T3 cells co-expressing CD59 and DAF are remarkably resistant to homologous complement attack whereas pcDNA3 control cells are susceptible to lysis. Fig. 5 showed that CD59 or DAF alone appear sufficient to protect the cells from limited complement attack and CD59 antigen appeared to be of more importance for cell survival in the face of complement attack, or alternatively that the expression level of hCD59 was higher than that of hDAF on the surface of NIH/3T3 cells. Our results indicate that coexpression of two proteins are more effective in limiting complement-dependent cytolysis than either protein being expressed alone on the surface of host cells. Hence, the recombinant construct exerts functional advantages over DAF and CD59 in the host cells. Gene transfer with a single vector, as used in our study, would be an effective strategy for the multiple and synchronous expression of the native hCRPs in transgenic animals, which has potential clinical use in preventing hyperacute rejection of transplanted organs. The results encourage us to generate double transgenic animals expressing hCD59 and $\mathrm{hDAF}$ for further analyzing and treating xenograft rejection in xenotransplantation.
Acknowledgments This work was supported by the grant from the National High-Technology Research and Development 863-Program of China (No.2001AA216071). We also thank Dr. J. Huang for her valuable suggestions and Miss Anjana Patel for helpful editing the manuscript.

\section{References}

Brodbeck, W. G., Mold, C., Atkinson, J. P. and Medof, M. E. (2000) Cooperation between decay-accelerating factor and membrane cofactor protein in protecting cells from autologous complement attack. J. Immunol. 165, 3999-4006.

Brooimans, R. A., VAN Wieringen, P. A., Vanes, L. A. and Daha, M. R. (1992) Relative roles of decay-accelerating factor, membrane cofactor protein, and CD59 in the protection of human endothelial cells against complement-mediated lysis. Eur. J. Immunol. 22, 3135-3140.

Byrne, G. W., McCurry, K. R., Kagan, D., Quinn, C., Martin, M. J., Platt, J. L. and Logan, J. S. (1995) Protection of xenogeneic cardiac endothelium from human complement by expression of CD59 or DAF in transgenic mice. Transplantation 60, 11491156.

Byrne, G. W., McCurry, K. R., Martin, M. J., McClellan, S. M., Platt, J. L. and Logan, J. S. (1997) Transgenic pigs expressing human CD59 and decay-accelerating factor produce an intrinsic barrier to complement-mediated damage. Transplantation 63, 149-155.

Calne, R. Y. (1970) Organ transplantation between widely disparate species. Transplant Proc. 2, 550-556.

Caras, I. W., Davitz, M. A., Rhee, L., Weddell, G., Martin, D. W. Jr. and Nussenzweig, V. (1987) Cloning of decay-accelerating factor suggests novel use of splicing to generate two proteins. Nature 325, 545-549.

Chen, R. H., Naficy, S., Logan, J. S., Diamond, L. E. and Adams, D. H. (1999) Hearts from transgenic pigs constructed with CD59/DAF genomic clones demonstrate improved survival in primates. Xeno. 6, 194-200.

Cowan, P. J., Aminian, A., Barlow, H., Brown, A. A., Chen, C. G., Fisicaro, N., Francis, D. M., Goodman, D. J., Han, W., Kurek, M., Nottle, M. B., Pearse, M. J., Salvaris, E., Shinkel, T. A., Stainsby, G. V., Stewart, A. B. and d'Apice, A. J. (2000) Renal xenografts from triple-transgenic pigs are not hyperacutely rejected but cause coagulopathy in non-immunosuppressed baboons. Transplantation 69, 2504-2515.

Cozzi, E., Bhatti, F., Schmoeckel, M., Chavez, G., Smith, K. G., Zaidi, A., Bradley, J. R., Thiru, S., Goddard, M., Vial, C., Ostlie, D., Wallwork, J., White, D. J. and Friend, P. J. (2000) Long-term survival of nonhuman primates receiving lifesupporting transgenic porcine kidney xenografts. Transplantation 70, 15-21.

Diamond, L. E., McCurry, K. R., Martin, M. J., McClellan, S. B., Oldham, E. R., Platt, J. L. and Logan, J. S. (1996) Characterization of transgenic pigs expressing functionally active human CD59 on cardiac endothelium. Transplantation 61, 1241-1249.

Fodor, W. L., Williams, B. L., Matis, L. A., Madri, J. A., Rollins, 
S. A., Knight, J. W., Velander, W. and Squinto, S. P. (1994) Expression of a functional human complement inhibitor in a transgenic pig as a model for the prevention of xenogeneic hyperacute organ rejection. Proc. Natl. Acad. Sci. USA 91, 11153-11157.

Fodor, W. L., Rollins, S. A., Guilmette, E. R., Setter, E. and Squinto, S. P. (1995) A novel bifunctional chimeric complement inhibitor that regulates $\mathrm{C} 3$ convertase and formation of the membrane attack complex. J. Immunol. 155, 4135-4138.

Gou, D. M., Huang, J., Li, W. X., Mao, X., Jiang, D. H., Hua, W. J. and Fan, J. H. (1999) Construction of early human embryo cDNA libraries and screening objective genes. Acta. Biochimica. Biophysica. Sinica. 31, 643-647.

Huang, J., Gou, D. M., Zhen, C. Y., Jiang, D. H., Mao, X., Li, W. X., Chen, S. and Cai, C. C. (2001) Protection of xenogeneic cells from human complement-mediated lysis by the expression of human DAF, CD59 and MCP. FEMS Immunol. Med. Microbiol. 31, 203-209.

Iwata, K., Seya, T., Ariga, H. and Nagasawa, S. (1994) Expression of a hybrid complement regulatory protein, membrane cofactor protein decay accelerating factor on Chinese Hamster Ovary. $J$. Immunol. 152, 3436-3444.

Laemmli, U. K. and Favre, M. (1970) Cleavage of structural proteins during the assembly of the head of bacteriophage T4. Nature 227, 680-685.

Lambrigts, D., Sachs, D. H. and Cooper, D. K. (1998) Discordant organ xenotransplantation in primates: world experience and current status. Transplantation 66, 547-561.

Lavitrano, M., Bacci, M. L., Forni, M., Lazzereschi, D., Di Stefano, C., Fioretti, D., Giancotti, P., Marfe, G., Pucci, L., Renzi, L., Wang, H., Stoppacciaro, A., Stassi, G., Sargiacomo, M., Sinibaldi, P., Turchi, V., Giovannoni, R., Della Casa, G., Seren, E. and Rossi, G. (2002) Efficient production by spermmediated gene transfer of human decay accelerating factor (hDAF) transgenic pigs for xenotransplantation. Proc. Natl. Acad. Sci. USA 99, 14230-14235.

Medof, M. E., Lublin, D. M., Holers, V. M., Ayers, D. J., Getty, R. R., L eykam, J. F., Atkinson, J. P. and Tykocinski, M. L. (1987) Cloning and characterization of cDNAs encoding the complete sequence of decay-accelerating factor of human complement. Proc. Natl. Acad. Sci. USA 84, 2007-2011.

Menoret, S., Plat, M., Blancho, G., Martinat-Botte, F., Bernard, P., Karam, G., Tesson, L., Renaudin, K., Guillouet, P., Weill, B., Chereau, C., Houdebine, L. M., Soulillou, J. P., Terqui, M. and Anegon, I. (2004) Characterization of human CD55 and CD59 transgenic pigs and kidney xenotransplantation in the pig-tobaboon combination. Transplantation 77, 1468-1471.

Meri, S., Morgan, B. P., Davies, A., Daniels, R. H., Olavesen, M. G., Waldmann, H. and Lachmann, P. J. (1990) Human protectin (CD59), an 18,000-20,000 MW complement lysis restricting factor, inhibits C5b-8 catalysed insertion of $\mathrm{C} 9$ into lipid bilayers. Immunology 71, 1-9.

Nagahama, M., Shiraishi, M., Oshiro, T. and Muto, Y. (2000) Adenovirus-mediated triple gene transfer of human complement regulating proteins to the porcine endothelial cell. Transplant Proc. 32, 2511-2513.

Nagahama, M., Shiraishi, M., Taira, K., Ohshiro, T. and Muto, Y. (2001) Adenovirus-mediated gene transfer of triple human complement regulating proteins in xenogeneic rat liver perfusion. Transplant Proc. 33, 779-780.

Nagahama, M., Shiraishi, M., Oshiro, T., Taira, K., Sugawa, H., Nozato, E., Nomura, H., Nagamine, M. and Muto, Y. (2002) Adenovirus-mediated gene transfer of triple human complement regulating proteins (DAF, MCP and CD59) in the xenogeneic porcine-to-human transplantation model. Part I: in vitro assays using porcine aortic endothelial cells. Transpl. Int. 15, 205-211.

Nicholson-Weller, A., Burge, J., Fearon, D. T., Weller, P. F. and Austen, K. F. (1982) Isolation of a human erythrocyte membrane glycoprotein with decay accelerating activities on C3 convertases of the complement system. J. Immunol. 129, 184-189.

Platt, J. L., Fischel, R. J., Matas, A. J., Reif, S. A., Bolman, R. M. and Bach, F. H. (1991) Immunopathology of hyperacute xenograft rejection in a swine-to-primate model. Transplantation 52, 214-220.

Rollins, S. A. and Sims, P. J. (1990) The complement inhibiting activity of CD59 resides in its capacity to block incorporation of C9 into membrane C5b-9. J. Immunol. 144, 3478-3483.

Sambrook, J., Fritsch, E. F. and Maniatis, T. (1989) Molecular Cloning: A Laboratory Manual, 2nd ed. Cold Spring Harbor Laboratory Press, NewYork, USA.

Shiraishi, M., Oshiro, T., Nozato E., Nagahama M., Taira K., Nomura H., Sugawa, H. and Muto, Y. (2002) Adenovirusmediated gene transfer of triple human complement regulating proteins (DAF, MCP and CD59) in the xenogeneic porcine-tohuman transplantation model. Part II: xenogeneic perfusion of the porcine liver in vivo. Transpl. Int. 15, 212-219.

Towbin, H., Staehlin, T. and Gordon, J. (1979) Electrophoretic transfer of proteins from polyacrylamide gels to nitrocellulose sheets: procedures and some applications. Proc. Natl. Acad. Sci. USA 76, 4350-4354. 